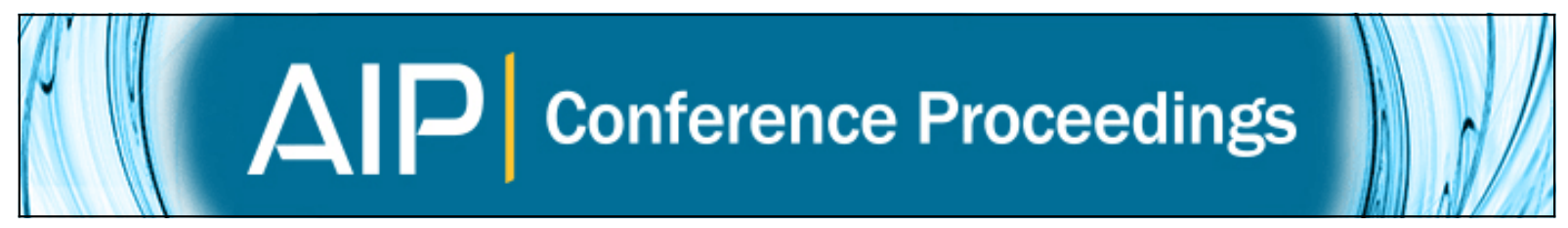

\title{
Molecular simulation of 3D turbulent channel flow
}

Dandan Zeng, Fengquan Zhong, and Jing Fan

Citation: AIP Conference Proceedings 1628, 1349 (2014); doi: 10.1063/1.4902748

View online: http://dx.doi.org/10.1063/1.4902748

View Table of Contents: http://scitation.aip.org/content/aip/proceeding/aipcp/1628?ver=pdfcov

Published by the AIP Publishing

\section{Articles you may be interested in}

Finite volume and finite element methods applied to 3D laminar and turbulent channel flows

AIP Conf. Proc. 1637, 607 (2014); 10.1063/1.4904630

3D RANS-RSTM numerical simulation of channel turbulent particulate flow with wall roughness

AIP Conf. Proc. 1558, 1067 (2013); 10.1063/1.4825690

Numerical simulation of reciprocating turbulent flow in a plane channel

Phys. Fluids 21, 095106 (2009); 10.1063/1.3225145

Properties of d-and k-type roughness in a turbulent channel flow

Phys. Fluids 19, 125101 (2007); 10.1063/1.2821908

Numerical simulation of pulsating turbulent channel flow

Phys. Fluids 13, 1367 (2001); 10.1063/1.1359766 


\title{
Molecular Simulation of 3D Turbulent Channel Flow
}

\author{
Dandan Zeng, Fengquan Zhong and Jing Fan \\ State Key Laboratory of High Temperature Gas Dynamics, Institute of Mechanics, \\ Chinese Academy of Sciences, Beijing 100190, China
}

\begin{abstract}
The diffusive information preservation (D-IP) method is utilized to simulate three-dimensional turbulent channel flow. The Knudsen number and Reynolds number based on the channel half-width and mean velocity are $5 \square 10^{-5}$ and 2800, respectively. The averaged velocity profile and the higher order turbulent statistics obtained by D-IP agree well with the DNS results given by Kim, Moin and Moser. Turbulent mixing length and turbulent viscosity obtained by the present results based on kinetic analogy are found to be comparable with the classic theory of Prandtl's mixing length and Boussinesq eddy viscosity.
\end{abstract}

Key words: molecular simulation, turbulent flow, three dimensional channel, D-IP method, kinetic analogy.

PACS: 02.70. Ns, $47.27 . n d$

\section{INTRODUCTION}

Turbulence is a fundamental issue characterized by multi-scale phenomena from macroscopic to molecular levels. It is unsolved completely but quite important in many engineering applications. Turbulent numerical studies are mostly based on continuum equations, such as RANS, LES \& DNS [1-3]. Molecular schemes such as the direct simulate Monte Carlo (DSMC) method, though widely applied to rarefied gas flow problems [4-7] and straightforward in principle for turbulence flows, are not feasible in practice for turbulent flows due to the statistical scatter and the rigorous restriction of temporal and spatial size. To solve the problem, the D-IP method was recently developed [8] that may be viewed as a combination of the diffusion approach [9] and the information preservation (IP) method [10]. The main idea of D-IP is to track the motion of a simulated molecule from the diffusive standpoint, and obtain the flow velocity and temperature through sampling and averaging the IP quantities. The D-IP method was validated through simulating benchmark problems [8], such as Couette flows, Poiseuille flows, Rayleigh flows and square cavity flows with $\mathrm{Kn} \sim 10^{-3}-10^{-4}$, and the D-IP calculations were shown not only accurate but also efficient in comparison with those using DSMC.

There is a great interest to extend the D-IP method to turbulence in order to understand the microscopic mechanism behind complicated flow patterns. A successful attempt for decaying and forced isotropic turbulences has been made by Fei et al. [11] The present paper aims to calculate turbulent channel flows using D-IP. As a type of wall turbulent flows, the turbulent channel flows often occur in natural processes and engineering applications. To validate the D-IP method, we employ the same computational parameters as those used in the DNS calculation of Kim, Moin \& Moser [12] where the Reynolds number (Re) based on the mean velocity $U_{m}$ and channel half width $\delta$ was 2800 . The averaged velocity profile and the higher order turbulent statistics obtained by D-IP and DNS will be compared with each other. Furthermore, the similarities and differences between molecular thermal motion and turbulent fluctuation will be investigated.

\section{METHODOLOGIES}

Figure 1 shows the flow geometry and the coordinate system, where the channel half width $\delta=2 \times 10^{4} \lambda_{m}\left(\lambda_{m}\right.$ : molecular mean free path), and the streamwise and spanwise lengths are $2 \pi \delta$ and $\pi \delta$, respectively. The computational domain is divided into $64 \times 96 \times 64$ cells that are non-uniform only in the y direction

$$
y_{i}=\frac{\delta}{\tanh b_{g}} \tanh \left[b_{g}\left(\frac{2 i}{N}-1\right)\right], b_{g}=2.0, i=0,1, \ldots, N .
$$

Proceedings of the 29th International Symposium on Rarefied Gas Dynamics AIP Conf. Proc. 1628, 1349-1355 (2014); doi: 10.1063/1.4902748 (C) 2014 AIP Publishing LLC 978-0-7354-1265-1/\$30.00 


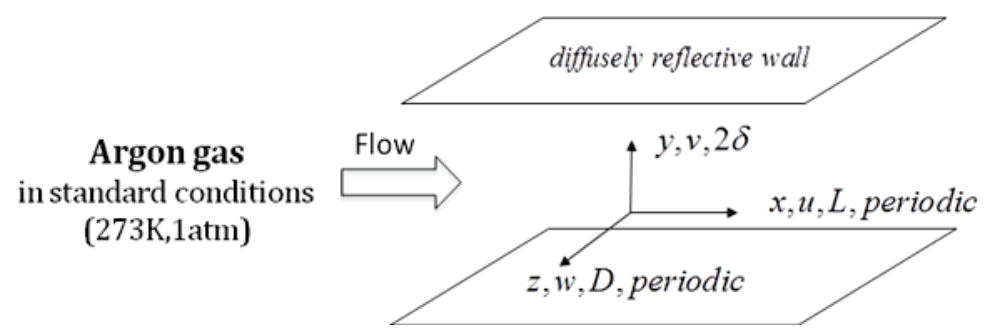

FIGURE 1. Schematic of turbulent channel flow and computational conditions.

The boundary conditions are periodic in the $x$ and $z$ directions but fully diffusive reflecting on the channel walls. The weighting factor of simulated molecules is proportional to the width of a cell in the $y$ direction. Initially, 100 simulated molecules are assigned to each cell, and their velocities are generated according to the local Maxwellian velocity distribution with the mean velocities equaling to the sum of the local laminar solution and random perturbations. The latter magnitudes in the three directions are all set to be $20 \%$ of the laminar solution. The time step is $50 \tau_{c}\left(\tau_{c}\right.$ : molecular mean collision time), about $1.7 \times 10^{-4} \delta / U_{m}$.

\section{Molecular Motion}

In the D-IP method [8], if $u_{I P, i}$ is the IP velocity of a simulated molecule, $\Delta r_{i}^{D}$ the diffusive drift, then molecular motion

$$
r_{i}(t+\Delta t)=r_{i}(t)+u_{I P, i} \times \Delta t+\Delta r_{i}^{D}
$$

According to the Chandrasekhar solution of the Langevin equation [13], we have

$$
\begin{gathered}
\Delta r_{i}^{D}=\frac{c_{i}(t)}{\zeta}\left(1-e^{-\zeta \Delta t}\right)+\sqrt{F} \theta_{1, i}, \\
c_{i}(t+\Delta t)=c_{i}(t) e^{-\zeta \Delta t}+\sqrt{\frac{H^{2}}{F}} \theta_{1, i}+\sqrt{G-\frac{H^{2}}{F}} \theta_{2, i}, \\
F=\frac{k_{B} T}{m \zeta^{2}}\left(4 e^{-\zeta \Delta t}-e^{-2 \zeta \Delta t}+2 \zeta \Delta t-3\right), \\
G=\frac{k_{B} T}{m}\left(1-e^{-2 \zeta \Delta t}\right), \\
H=\frac{k_{B} T}{m \zeta}\left(1-e^{-\zeta \Delta t}\right)^{2},
\end{gathered}
$$

where $c_{i}$ is the thermal velocity of the simulated molecule. $\zeta=k_{B} T / m D, k_{B}$ the Boltzmann constant, $T$ the temperature, $m$ the molecular mass, $D$ the diffusion coefficient, and $\theta_{1, i}$ and $\theta_{2, i}$ random numbers that obeys the standard normal distribution.

\section{Dissipation of the IP Velocity and Temperature}

If $u_{I P, j}^{i, c}$ and $u_{I P, j}^{i, a}$ are the IP velocities in the j-direction of simulated molecule i before and after the motion and collisions during a time step, respectively, then

with

$$
u_{I P, j}^{i, a}=u_{I P, j}^{i, c}+w\left(\frac{1}{N} \sum_{k \leq N} u_{I P, j}^{k, c}-u_{I P, j}^{i, c}\right)
$$




$$
\begin{gathered}
w=\frac{2 D \Delta t}{\Delta x_{s}^{2}}+\frac{2 D \Delta t}{\Delta y_{s}^{2}}+\frac{2 D \Delta t}{\Delta z_{s}^{2}}, \\
\left|r_{1}^{k}-r_{1}^{i}\right| \leq \Delta x_{s},\left|r_{2}^{k}-r_{2}^{i}\right| \leq \Delta y_{s},\left|r_{3}^{k}-r_{3}^{i}\right| \leq \Delta z_{s} .
\end{gathered}
$$

In the present calculations, $\Delta x_{s}=0.25 \Delta x, \Delta y_{s}=0.25 \Delta y$, and $\Delta z_{s}=0.25 \Delta z$

A similar scheme can be used to calculate the dissipative processes of IP temperature. The total momentum and energy are required to be conserved in the process.

\section{Macroscopic Velocity and Temperature}

If $\mathrm{u}_{\mathrm{i}, \mathrm{k}}$ and $\mathrm{T}_{\mathrm{k}}$ are the macroscopic velocity and temperature of cell $\mathrm{k}$ in a flow field, then

$$
u_{i, k}=\frac{1}{N_{k}} \sum_{j=1}^{N_{k}} u_{I P, i, j}, \quad T_{k}=\frac{1}{N_{k}} \sum_{j=1}^{N_{k}} T_{I P, j},
$$

where $\mathrm{N}_{\mathrm{k}}$ is the total number of the simulated molecules sampled in cell $\mathrm{k}$.

\section{Effects of Pressure Gradient and External Force $F_{i}$}

Density of the field is updated via continuum Eq. (12), the effect of pressure and external force to the velocity and temperature can be expressed by Eqs. (13) and (14).

$$
\begin{gathered}
\frac{\partial(n m)}{\partial t}+\frac{\partial\left(n m u_{j}\right)}{\partial x_{j}}=0 \\
\frac{\partial}{\partial t}\left(n m u_{I P, i}\right)=-\frac{\partial p}{\partial x_{i}}+n m F_{i}, \\
\frac{\partial}{\partial t}\left(n m C_{v} T_{I P}\right)=-p \frac{\partial u_{j}}{\partial x_{j}}
\end{gathered}
$$

where $n$ is the number density of molecules, $C_{v}$ the specific heat at constant volume, and $p=n k_{B} T$.

\section{Simplifications}

It is well known that the Mach number (Ma) is related to Kn and Re, approximately, $M a \approx 0.7 \operatorname{Re} K n$. From this relation we know Ma is about 0.1 in the present condition. Consequently, the isothermal assumption is invoked and the compressible effect is negligible. Based on the above analysis, the calculation can be expressed by Eqs. (2), (8), (12) and (13).

The calculation procedures each time step are as follows: i) simulated molecules move according to Eq. (2); ii) the velocity field is updated according to Eq. (11); iii) exchange the IP velocities between molecules following Eq. (8); iv) solve Eqs. (12) and (13) to update the corresponding macroscopic quantities.

\section{VALIDATION}

The D-IP calculation consists of two stages. The first stage begins from the initial perturbed laminar flow state until it reaches a steady state in statistic sense. The steady state can be identified by examining the skin friction coefficient $c_{f}=2 u_{\tau}^{2} / U_{m}^{2}$, where $u_{\tau}^{2}=v_{m} \times \partial U /\left.\partial y\right|_{w}, v_{m}$ is the gas kinetic viscosity, $U=\langle u\rangle,\langle\rangle$ denotes an average over $\mathrm{x}, \mathrm{z}$ and $\mathrm{t}$, and the subscript $\mathrm{w}$ denotes at the channel walls. The first stage ends when $c_{f}=7.96 \times 10^{-3}$ that compares well the corresponding value of $8.18 \times 10^{-3}$ in literature [12]. In the second stage, the velocity and velocity fluctuations are obtained through compared to the previously published DNS result. 
Figure 2 compares the averaged velocity profiles obtained by D-IP and DNS, where the superscript " + " denotes dimensionless quantities scaled by wall units, specifically, the friction velocity $u_{\tau}$ and viscous lengthscale $v_{m} / u_{\tau}$ for velocity and length respectively. The DNS results came from Kim et al. [12]. The viscous sublayer and logarithmic region are clearly observed in the velocity profile of D-IP that agrees well with Kim's data.

Turbulent fluctuation intensities in the three directions, Reynolds shear stress, and skewness and flatness are illustrated and compared with DNS results in Figs.3 (a), (b) and (c) respectively. The D-IP profiles are generally in good agreement with those of Kim et al. [12], except for the small discrepancies of turbulence fluctuation velocities near the wall.

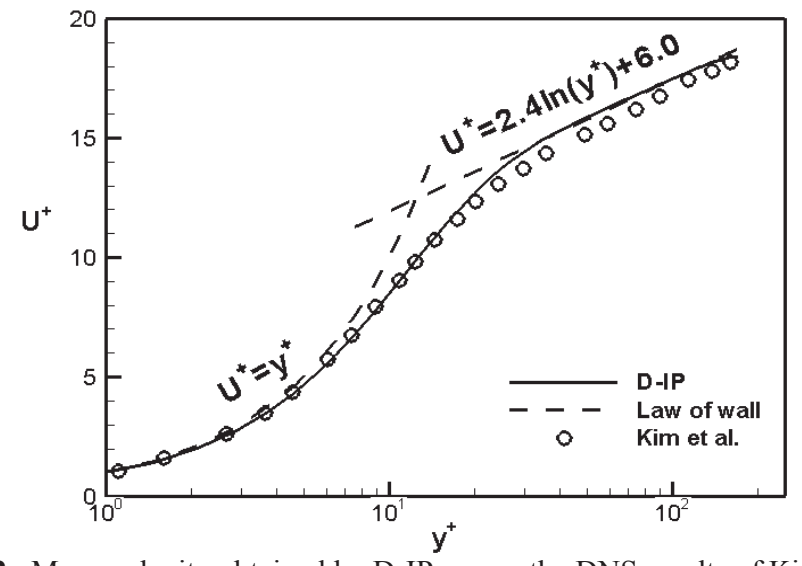

FIGURE 2. Mean velocity obtained by D-IP versus the DNS results of Kim et al. [12].

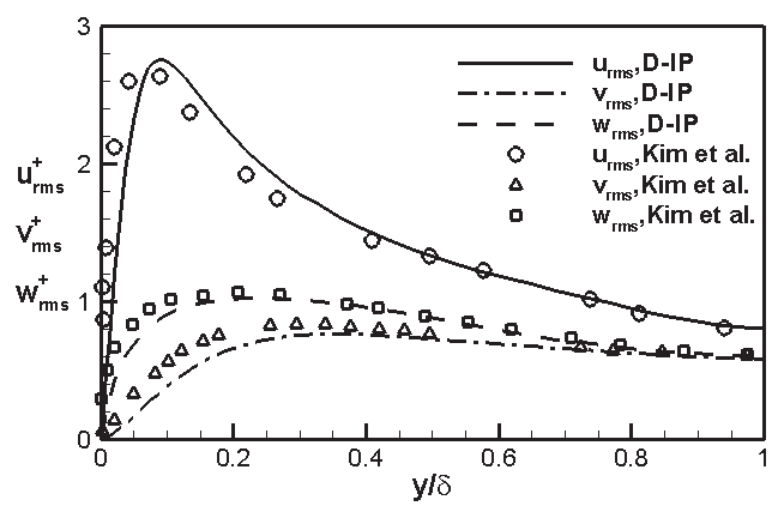

(a) Turbulent fluctuation velocity

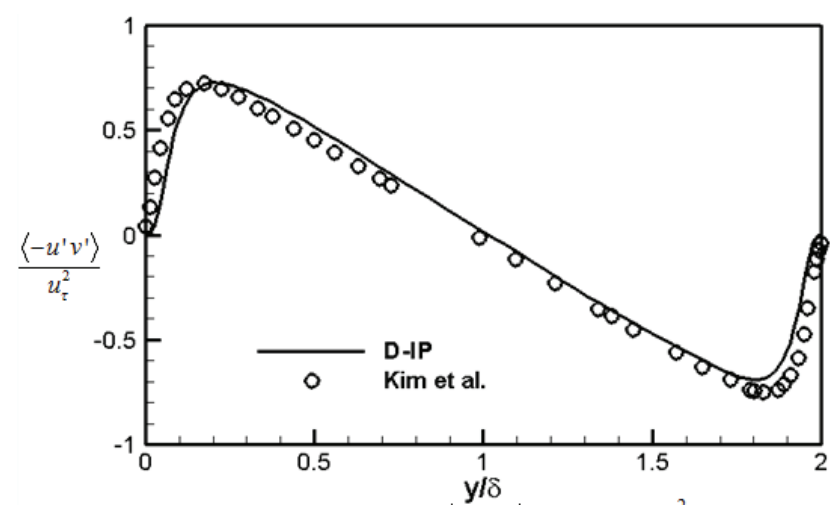

(b) Reynolds shear stress

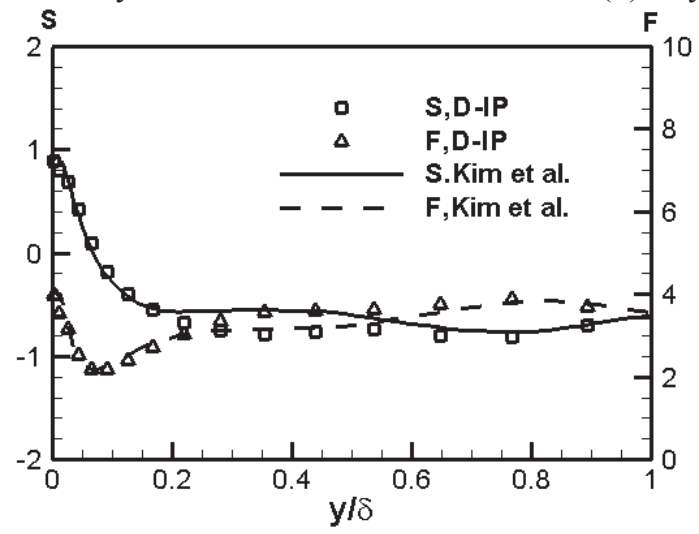

(c) Skewness (S) and flatness (F)

FIGURE 3. Turbulence fluctuation velocities, Reynolds shear stress, skewness and flatness obtained by D-IP versus the DNS results of Kim et al. [12]. 


\section{MOLECULAR THERMAL MOTION VERSUS TURBULENT FLUCTUATION}

Turbulent fluctuation is compared with molecular thermal motion from four aspects: the autocorrelation functions and their characteristic time, the root mean square velocity, mean free path and turbulent mixing length, and viscosity.

The normalized autocorrelation function of molecular thermal motion $K_{m}(t)$ may be written as

$$
K_{m}(t)=\frac{\left\langle c_{x}{ }^{\prime}(t) c_{x}{ }^{\prime}(0)\right\rangle}{k_{B} T / m}=\exp \left[-2 t /\left(3 \tau_{c}\right)\right] .
$$

If the characteristic time of the autocorrelation of molecular thermal motion is defined as $K_{m}\left(\tau_{a}^{m}\right)=1 / e$, then $\tau_{\mathrm{a}}^{m}=1.5 \tau_{c}$.

Similarly, the autocorrelation function of turbulent fluctuation velocity $K_{t}\left(t, y_{k}\right)$ is defined as

$$
K_{t}\left(t, y_{k}\right)=\frac{1}{N_{k}} \sum_{i=1}^{N_{k}} \frac{v_{I P, i}^{k}(t) v_{I P, i}^{k}(0)}{v_{I P, r m s}^{j}(t) v_{I P, r m s}^{k}(0)}, \quad y_{j}<y_{i}^{k}(t)<y_{j+1},
$$

where $v_{I P, i}^{k}$ is the fluctuation velocity of simulated molecule i initially in layer $\mathrm{k}$ in the normal direction, $v_{I P, r m s}^{j}$ is the root mean square velocity obtained by all the simulated molecules in layer $\mathrm{j}$ where simulated molecule $\mathrm{i}$ located at $\mathrm{t}$.

If the characteristic time of turbulent fluctuation velocity $\tau_{a}^{t}$ is defined as $K_{t}\left(\tau_{a}^{t}\right)=1 / e$, then a relation of $\tau_{a}^{t}$ to the distance from the channel can be obtained from the D-IP results shown in Fig. 4. Figure 5 presents the relation that approximately linearly increases in the central region, where the superscript "+" denotes the dimensionless time normalized by $v_{m} / u_{\tau}^{2}$.

The root mean square velocity $v_{r m s}^{m}$ of molecular thermal motion is simply $\sqrt{k_{B} T / m}$. It is a constant across the channel under the isothermal condition. For turbulence, we define the r.m.s. velocity $v_{r m s}^{t}$ as the r.m.s. turbulent fluctuation velocity in normal direction.

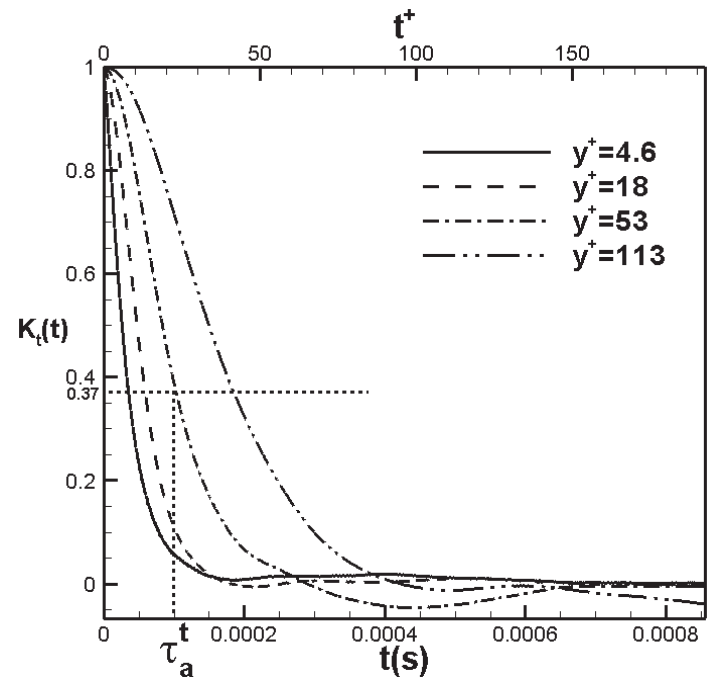

FIGURE 4. Autocorrelation function of turbulent fluctuation velocity at different distances from the channel wall.

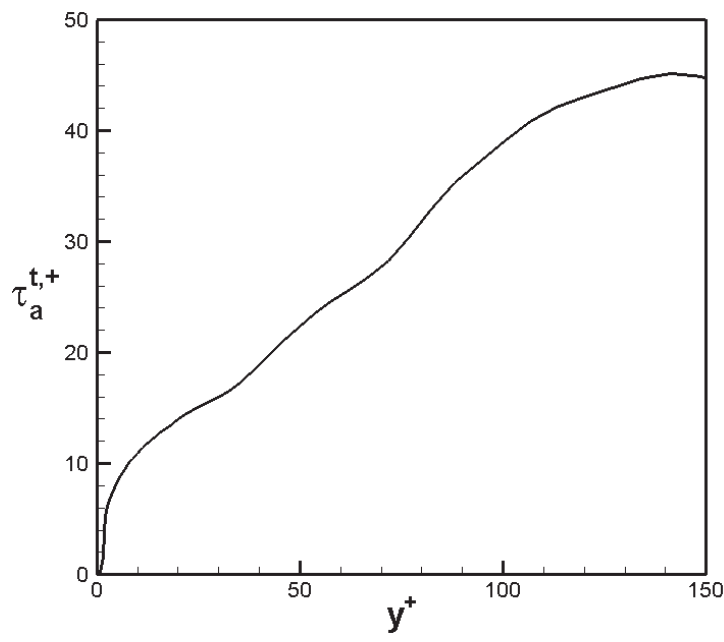

FIGURE 5. Characteristic time of the autocorrelation of turbulent fluctuation velocity versus the distance from the wall. 
According to kinetic theory [4], the molecular mean free path and viscosity may be related to $\tau_{\mathrm{a}}^{m}$ and $v_{r m s}^{m}$ as follows

$$
\lambda_{m}=\frac{4}{3} \sqrt{\frac{2}{\pi}} \times \tau_{a}^{m} v_{r m s}^{m}, \quad v_{m}=\frac{5}{6} \tau_{a}^{m}\left(v_{r m s}^{m}\right)^{2}
$$

Based on a kinetic analogy between molecular thermal motion and turbulent fluctuation velocity, the turbulent mixing length $\ell_{m}$ and the turbulence viscosity $v_{t}^{k}$ have the following expressions

$$
\begin{gathered}
\ell_{m}=\frac{4}{3} \sqrt{\frac{2}{\pi}} \times \tau_{a}^{t} v_{r m s}^{t}, \\
v_{t}^{k}=\frac{5}{6} \tau_{a}^{t}\left(v_{r m s}^{t}\right)^{2} .
\end{gathered}
$$

Figure 6 presents the turbulent mixing length across the channel given by Eq. (18), with $\tau_{a}^{t}$ and $v_{r m s}^{t}$ obatined by the D-IP calculation and shown in Figs. 5 and 2, respectively. It agrees well with the classical theory of Prandtl in the logarithmic region; in the central region, it tends to be flat rather than a linear increase with $\mathrm{y}$, which is more physically reasonable.

Figure 7 presents the turbulence viscosity given by Eq. (19) that is comparable with the Boussinesq eddy viscosity $v_{t}^{B}$. The latter is obtained by the relation $v_{t}^{B} d U / d y=-\left\langle u^{\prime} v^{\prime}\right\rangle$, with $d U / d y$ and $\left\langle u^{\prime} v^{\prime}\right\rangle$ obatined by the DIP calculation and shown in Figs. 1 and 2, respectively.

\section{CONCLUSION}

The D-IP method has been applied to a turbulent channel flow and compared in details with the DNS results of Kim, Moin \& Moser. The comparison validates the feasibility and accuracy for D-IP to simulate the typical wall turbulence. Based on the D-IP results, turbulence mixing length and viscosity are studied via kinetic analogy between molecular thermal motion and turbulent fluctuation velocity. The classic theory of turbulence such as the Prandtl mixing length and the Boussinesq eddy viscosity has been investigated in a kinetic view that is helpful to undertand them more deeply.

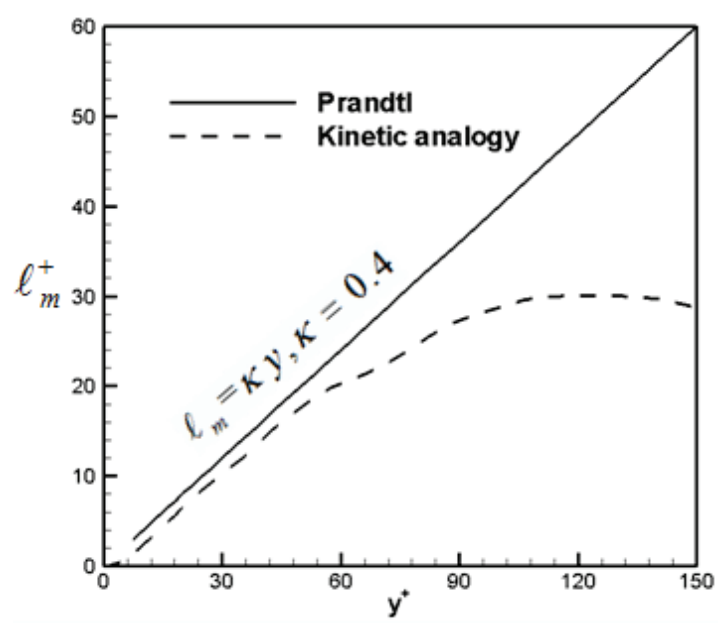

FIGURE 6. Turbulent mixing length versus the distance from the channel wall.

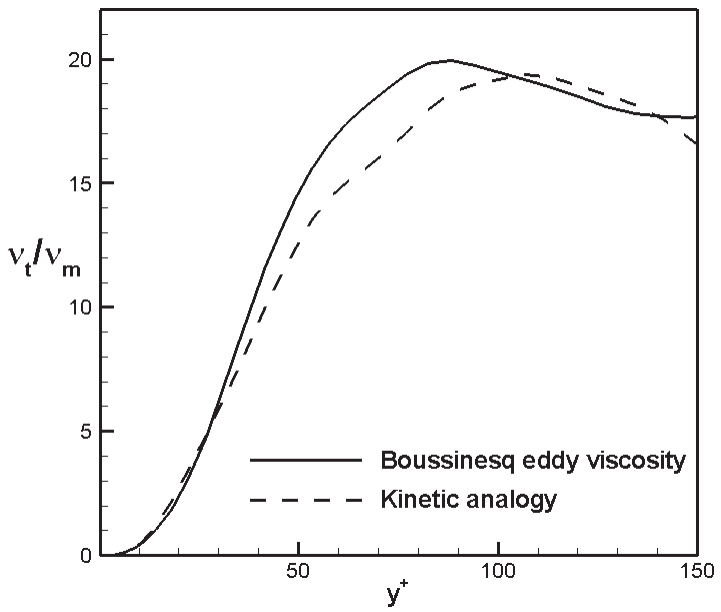

FIGURE 7. Normalized turbulent viscosity versus the distance from the channel wall. 


\section{REFERENCES}

1. P. R. Spalart, S. Deck, M. L. Shur, K. D. Squires, M. K. Strelets and A. Travin, Theor. Comput. Fluid Dyn. 20, 181-195 (2006).

2. S. Fu and L. Wang, Progress in Aerospace Sciences 58, 36-59 (2013).

3. P. Moin and K. Mahesh, Annu. Rev. Fluid Mech. 30, 539-578 (1998).

4. G.A. Bird, Molecular Gas Dynamics and the Direct Simulation Monte Carlo Method, Oxford: Clarendon Press, 1994.

5. M. S. Ivanov and S. F. Gimelshein, Annu. Rev. Fluid Mech. 30, 469-505 (1998).

6. E. S. Oran, C. K. Oh and Z. C. Cybyk, Annu. Rev. Fluid Mech. 30, 403-441 (1998).

7. J. Fan. Advances in Mechanics, 43: 185-201 (2013)

8. F. Fei and J. Fan, J. Comput. Phys. 243, 179-193(2013).

9. P. Jenny, M. Torrilhon and S. Heinz, J. Comput. Phys. 229, 1077-1098(2010).

10. J. Fan and C. Shen, J. Comput. Phys. 167, 393-412(2001).

11. F. Fei, J. Fan, C. X. Xu, Y. Song, Z. H. Liu, "Statistical Simulation of Decaying and Forced Homogeneous Isotropic Turbulence", presented at the 29th International Symposium on Rarefied Gas Dynamics, July 13-18, 2014, Xian, China.

12. J. Kim, P. Moin and R. Moser, J. Fluid Mech. 177, 133-166(1987).

13. S. Chandrasekhar, Rev. Mod. Phys. 15, 1-89 (1943). 